\title{
Aporte de la ecocardiografía tridimensional en el diagnóstico y manejo de la endocarditis infecciosa. Experiencia clínica
}

\author{
Julián Vega ${ }^{1}$, Luigi Gabrielli ${ }^{1}{ }^{2}$, Samuel Córdova ${ }^{1}$, María Cecilia Muñoz ${ }^{1}$, Paul McNab $^{1}$, \\ Rodrigo Saavedra ${ }^{1}$, Ricardo Zalaquett ${ }^{1}, 2$. \\ 1. División de Enfermedades Cardiovasculares. Escuela de Medicina. Pontificia Universidad \\ Católica de Chile. Santiago, Chile. \\ 2. Advanced Center for Chronic Diseases (ACCDiS), Escuela de Medicina. Pontificia Universidad \\ Católica de Chile. Santiago, Chile. \\ Sin apoyo financiero
}

El diagnóstico ecocardiográfico (eco) de la endocarditis infecciosa ha tenido una evolución vertiginosa, destacando el desarrollo de la ecocardiografía transesofágica (ETE), la imagen armónica y la ecocardiografía tridimensional (Eco 3D), tecnologías que han incrementado su capacidad diagnóstica. Presentamos una serie de endocarditis donde el Eco 3D tuvo un rol importante. CASO 1: Hombre de 18 años, antecedente remoto de artritis séptica y deterioro de capacidad funcional asociado a soplo de insuficiencia mitral. La ecocardiografía transtorácica (ETT) objetivó leve dilatación ventricular izquierda e insuficiencia mitral severa, el Eco 3D identificó una perforación circular en el velo anterior mitral, logrando la reparación valvular con un parche de pericardio. CASO 2. Hombre de 29 años, con absceso dental reciente, se presenta en shock séptico, el ETT y ETE mostraron una válvula aórtica bicúspide (VAB) con insuficiencia severa y un absceso del velo anterior mitral. El Eco 3D identificó una perforación del velo no rafial, se realizó un doble recambio valvular. CASO 3: Hombre de 61 años, con antecedente previo de endocarditis y VAB. Consulta por estado infeccioso. El ETT y ETE informaron insuficiencia aórtica moderada asociado a insuficiencia mitral severa, el eco 3D identificó prolapso del segmento P3 de la válvula mitral, ayudando a programar su reparación. CASO 4. Mujer de 78 años, con diagnóstico de endocarditis mitral protésica, el Eco 3D objetivó un absceso peri-protésico posterior de $16 \mathrm{~mm}$, siendo referida a cirugía.

El Eco 3D aporta información complementaria en diversos escenarios de la endocarditis, tales como: perforaciones valvulares, compromiso peri-anular, endocarditis protésica, optimizando su manejo. 


\section{Added value of three-dimensional echocardiography in the diagnosis and management of infective endocarditis. Clinical experience.}

The echocardiographic diagnosis of infective endocarditis has evolved in a vertiginous manner. Among its main hallmarks are; the development of transesophageal echocardiography (TEE), harmonics imaging and three-dimensional echocardiography (3D Echo), thereby enhancing its diagnosis capability. The following endocarditis cases revealed the usefulness of 3D Echo. CASE 1. 18 year-old male, past history of septic arthritis, refers decline in functional capacity associated with a mitral regurgitation murmur. Transthoracic echocardiography (TTE) pictured a mildly dilated left ventricle and severe mitral regurgitation. 3D Echo identified a circular perforation of the anterior mitral leaflet; successful mitral valve repair was accomplished. CASE 2. 29 year-old male, with recent dental abscess, presents in septic shock. TTE and TEE portrayed a bicuspid aortic valve (BAV) with severe regurgitation along with an abscess of the anterior mitral valve leaflet. 3D Echo showed a perforation of the non-fused aortic leaflet; double valve replacement was uneventfully performed. CASE 3. 61 year-old male, with remote history of endocarditis and BAV, evaluated with TTE and TEE due to sepsis, depicting moderate aortic regurgitation and $\mathrm{P} 3$ prolapse with rupture chordae and severe mitral regurgitation. Based on the 3D Echo data the mitral valve was repaired and the aortic valve replaced. CASE 4. 78 year-old woman with prosthetic mitral valve endocarditis, 3D Echo allowed complete visualization of a $16 \mathrm{~mm}$ diameter posterior abscess, being referred to surgery. Thus, 3D Echo has added value in several endocarditis scenarios such as; leaflet perforations, periannular involvement, prosthetic valve endocarditis, improving its management.

MesH (Key words): Endocarditis, echocardiography, three-Dimensional 


\section{Inroducción:}

El diagnóstico imagenológico de la endocarditis infecciosa y de sus complicaciones ha tenido una vertiginosa evolución, experimentando una total transformación desde el primer reporte ecocardiográfico de las vegetaciones valvulares por James Dillon en $1973^{1}$ hasta la actualidad. Dentro de sus principales hitos se pueden nombrar; a final de la década de 1980 el desarrollo de la ecocardiografía transesofágica (ETE), seguido de la incorporación de la imagen armónica y a comienzos de los años 2000 la llegada de la ecocardiografía tridimensional (Eco 3D). Esta tecnología permitió una integración espacial total de las estructuras cardíacas, aspecto notable para una técnica basada en ultrasonido. En consecuencia, la ecocardiografía ha conquistado un rol protagónico en las guías clínicas de endocarditis para todas las facetas de la enfermedad ${ }^{2}$; establecer diagnóstico de la patología y de sus complicaciones, determinar pronóstico, guiar el manejo y seguimiento. A continuación, presentamos una serie de casos e imágenes de pacientes con endocarditis infecciosa manejados en nuestra institución y donde el Eco 3D tuvo un importante rol.

CASO 1. Hombre de 18 años, caso reportado previamen$\mathrm{te}^{3}$, con antecedente remoto de artritis séptica de rodilla. Consulta por deterioro de la capacidad funcional durante los últimos 2 años. El examen físico pesquisa soplo de insuficiencia mitral. La ecocardiografía transtorácica (ETT) muestra leve dilatación ventricular izquierda con función sistólica conservada, asociado a insuficiencia mitral severa con jet de recorrido muy excéntrico, sin lograr esclarecer su etiología. Se efectuó un ETE (Figura 1, Panel A) obteniendo volúmenes en $3 \mathrm{D}$ de la válvula mitral, mediante "zoom 3D" y 3D en tiempo real, optimizando los parámetros para obtener $>12$ volúmenes por segundo (Figura 1, Panel B). La reconstrucción multiplanar (MPR) de la válvula mitral (Figura 1, Panel C) permitió identificar una perforación circular de $6 \times 6 \mathrm{~mm}$, localizada en el cuerpo del segmento medio del velo anterior mitral (A2), con margen de $3 \mathrm{~mm}$ hacía el borde libre del velo. Se evaluó en conjunto con el equipo de cirugía cardíaca, interpretando dicha lesión como una secuela de endocarditis. En base a los hallazgos del Eco 3D se estimó una alta posibilidad de reparación. La cirugía cardíaca corroboró lo descrito por el Eco 3D (Figura 2, Panel E y F), logrando la reparación valvular mediante un parche de pericardio (Figura 2, Panel D). La evolución fue satisfactoria y el ETE de control al año descarto insuficiencia valvular residual.

APORTE DEL ECO 3D: El Eco 3D tiene mejor rendi-
Figura 1. ETE, Eco 3D y reconstrucción multiplanar (MPR) de la válvula mitral.

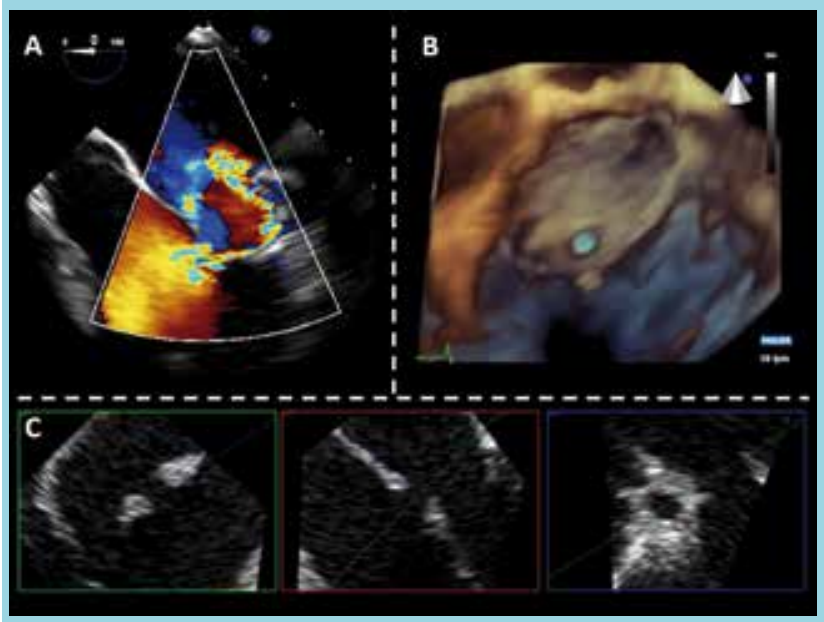

PANEL A: ETE medio esofágico a $0^{\circ}$ con Doppler color. Se observa un jet de regurgitación originado en A2 con una gran zona de PISA espontáneo (Nyquist de $60 \mathrm{~cm} / \mathrm{s}$ ) dirigido hacia el velo posterior. PANEL B. 3D en tiempo real de la válvula mitral, se ha recortado el velo posterior y se observa la perforación circular en el segmento medio del velo anterior mitral con un margen de $3 \mathrm{~mm}$ hacia el borde libre. PANEL C. Planos de la reconstrucción multiplanar (MPR) de la válvula mitral, en verde y rojo se observan planos ortogonales de la perforación que permiten su correcta medición: $6 \times 6 \mathrm{~mm}$. Información complementaria disponible en http://ecocardio.cl/pmitral.html

Figura 2. Hallazgos intraoperatorios y reparación valvular mitral.

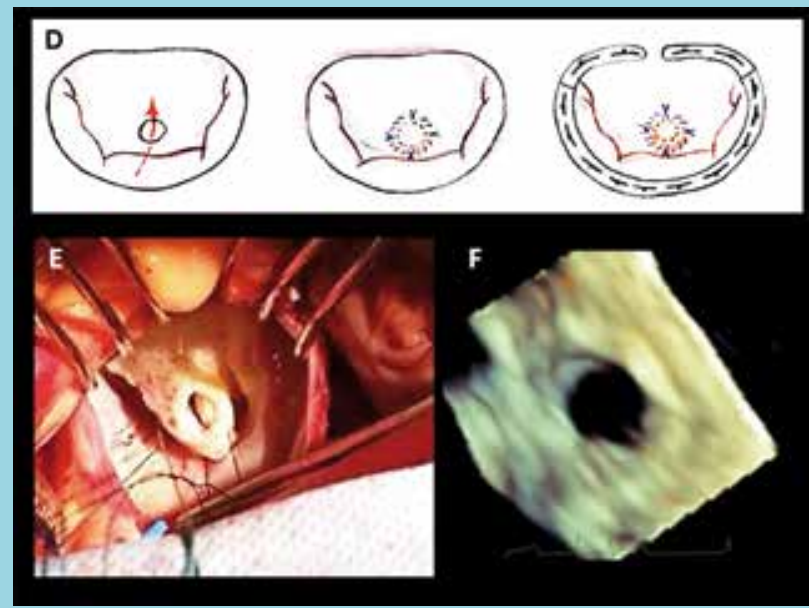

PANEL D: Esquema de la lesión y de su reparación con un parche de pericardio más anuloplastía con anillo.

PANEL E: Imagen del intraoperatorio demostrando la perforación circular del segmento A2.

PANEL F: Imagen 3D con zoom de la perforación.

Información complementaria disponible en

http://ecocardio.cl/pmitral.html 
Figura 3. Eco 3D de la válvula aórtica.

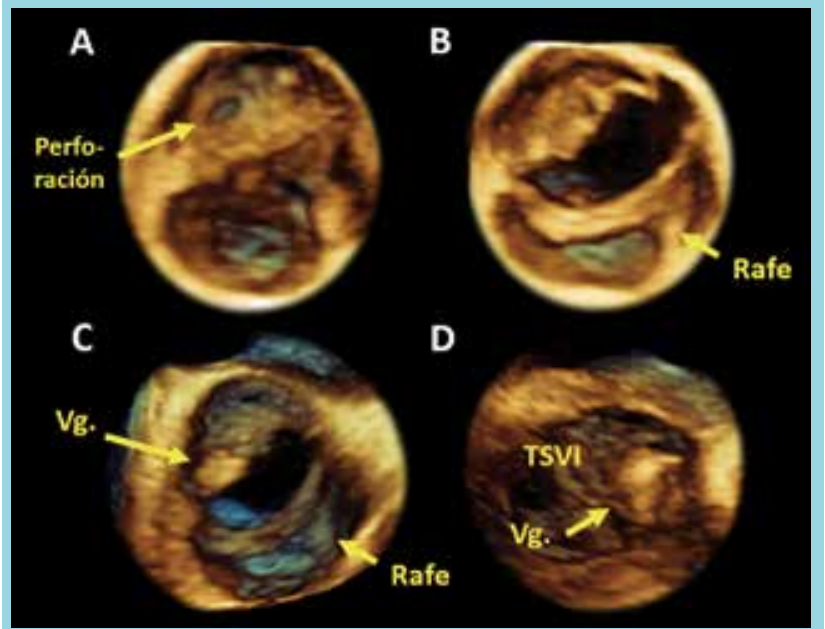

PANEL A. Eco 3D de la VA vista desde la cara aórtica en diástole, se observa una perforación circular en el velo no rafial. PANEL B. Eco 3D de la VA vista desde la cara aórtica en sístole, se identifica el rafe en posición posterior y la apertura en "ojal" de la válvula. PANEL C. Eco 3D de la VA vista desde la cara ventricular. Se identifica adherido al borde libre del velo no rafial una vegetación (vg.) que se observa en el PANEL D. Prolapsando al tracto de salida del ventrículo izquierdo (TSVI) en diástole.

TSVI: tracto de salida del ventrículo izquierdo. Vg: Vegetación.

Figura 4. Absceso de la válvula mitral.

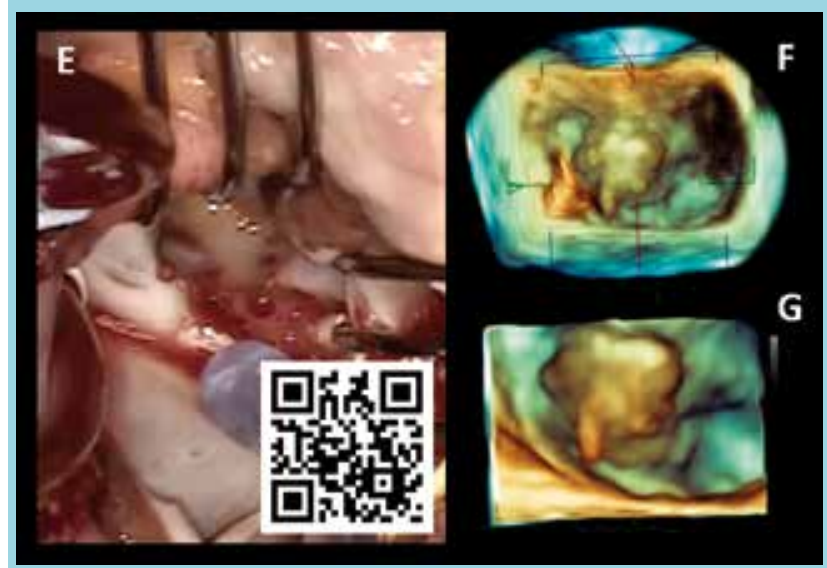

PANEL E. Imagen del intraoperatorio, visión desde un acceso transeptal ampliado, exponiendo la cara auricular de la válvula mitral que presenta un absceso en el velo anterior. PANEL F. Eco 3D de la válvula mitral "EN FACE" que permite delimitar el absceso y localizarlo sobre A1 y A2. PANEL G. Eco 3D con zoom sobre el absceso lo que otorga una mayor resolución temporal y espacial.

Video con información complementaria disponible escaneando el código QR de la imagen. miento para diagnosticar perforaciones valvulares, permitiendo además presentar la lesión valvular al equipo de cirugía desde una perspectiva familiar (vista "EN FACE" de la válvula mitral).

CASO 2. Hombre de 29 años, sin antecedentes mórbidos. Con terapia reciente por absceso dental, relata estado infeccioso de 1 mes de evolución. Consulta en regulares condiciones generales, taquicárdico, mal perfundido, el examen físico objetiva soplo de insuficiencia aórtica. El ETT y ETE muestran: ventrículo izquierdo de tamaño normal con aspecto hiperdinámico, válvula aórtica bicúspide por fusión de velos coronarianos, identificando una vegetación de $2 \mathrm{~cm}$ adherida al borde libre del velo no rafial, asociado a insuficiencia severa por un jet de recorrido excéntrico, cuyo mecanismo no fue posible establecer por ecocardiografía bidimensional (Eco 2D). La válvula mitral presentaba en su velo anterior hacia los segmentos A1 y A2 por su cara auricular una zona de engrosamiento heterogéneo de 16 x $11 \mathrm{~mm}$ compatible con un absceso, sin alteraciones funcionales al Doppler. El Eco 3D permitió aclarar el mecanismo de la insuficiencia aórtica, identificando una válvula bicúspide, con una perforación en el cuerpo del velo no rafial (Figura 3). Visualizando además una vegetación adherida al borde libre de dicho velo que prolapsaba hacia el tracto de salida en diástole (Figura 3, Panel C y D). Asimismo, el Eco 3D delimitó la localización y tamaño del absceso mitral (Figura 4, Panel F y G). La cirugía cardíaca confirmó los hallazgos descritos, realizando un doble recambio valvular con prótesis biológicas, con buena evolución postoperatoria.

APORTE DEL ECO 3D. El 3D permitió identificar el mecanismo de la insuficiencia aórtica (perforación) y delimitar el absceso mitral.

CASO 3. Hombre de 61 años, con antecedente de endocarditis tratada medicamente el año 2011, episodio en el que se diagnosticó una valvulopatía aórtica bicúspide con insuficiencia moderada. Consulta por 1 semana de deterioro de la capacidad funcional asociado a fiebre y calofríos. Se realiza ETT y ETE que informan; severa dilatación de cavidades cardíacas izquierdas con buena motilidad global del ventrículo izquierdo; válvula aórtica bicúspide por fusión de velos coronarianos, con pequeños elementos móviles en el borde libre de sus velos e insuficiencia aórtica severa por un jet de recorrido excéntrico. La válvula mitral presentaba un prolapso de su velo posterior hacia el segmento medial con una posible 


\section{Figura 5. Eco 3D del prolapso de P3.}

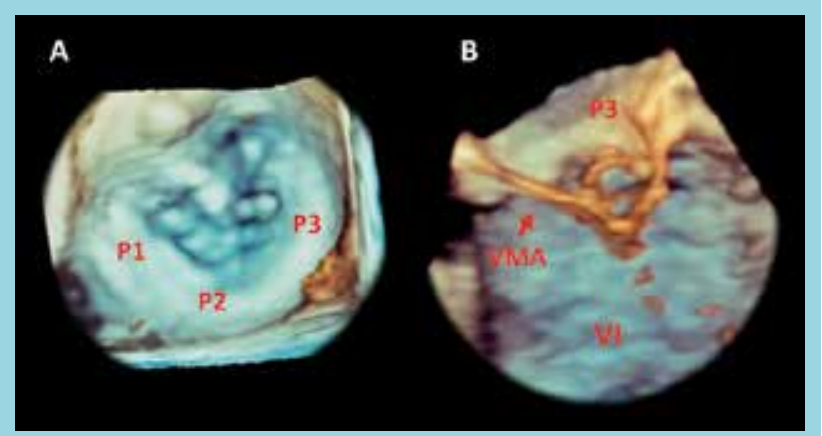

PANEL A. Eco 3D de la válvula mitral desde una visión "EN FACE" en sístole. Se observa el prolapso del segmento P3 asociado a una imagen de cuerda rota. PANEL B. Eco 3D de la válvula mitral desde una visión medial en sístole, se observa el prolapso en forma de "capucha" del segmento P3.

VI: Ventrículo izquierdo. VMA: Velo mitral anterior. P1: Segmento lateral del velo mitral posterior. P2: Segmento medio del velo mitral posterior. P3: Segmento medial del velo mitral posterior.

Figura 6. Eco 3D de la prótesis valvular mitral.

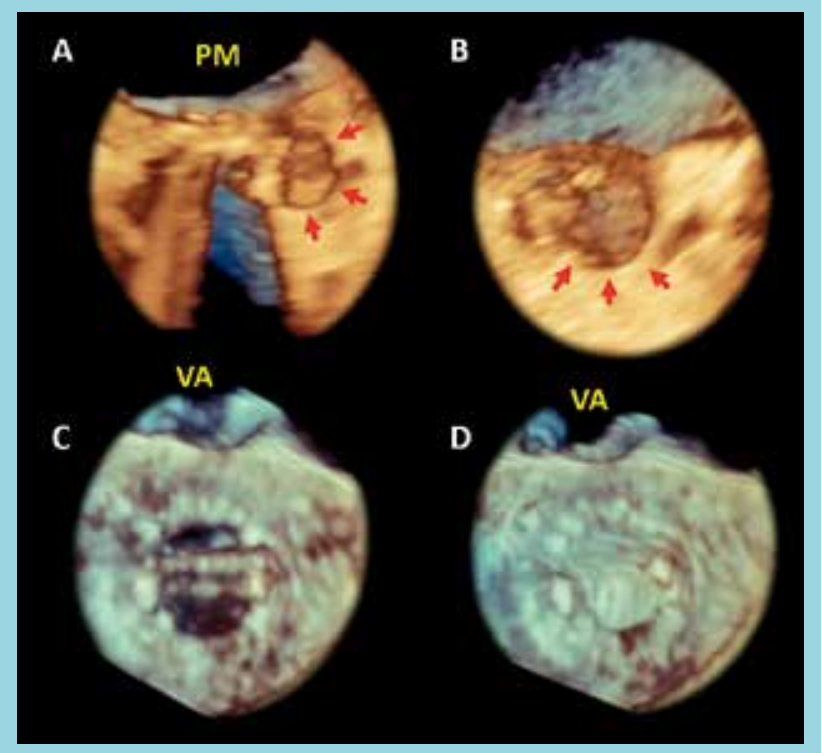

PANEL A: Eco 3D de la prótesis mitral, se ha recortado la prótesis desde su cara lateral observando la cavidad delimitada por el absceso (flecha roja). PANEL B: Eco 3D con zoom sobre el absceso peri-protésico. PANEL C. Prótesis mitral bivalva con adecuado funcionamiento de sus discos, apertura completa y simétrica en sístole y en el PANEL D. Se observa el adecuado cierre diastólico. PM: Prótesis mitral. VA: Válvula aórtica.

imagen de cuerda rota e insuficiencia severa. El Eco 3D permitió caracterizar de mejor forma la lesión, identificando una cuerda rota y el prolapso del segmento P3 que generaba una imagen tipo "capucha", que se encontraba perforada en su base, sitio desde donde se originaba la insuficiencia (Figura 5). Se realizó recambio valvular aórtico con prótesis biológica y apoyado por los hallazgos del Eco 3D, se decidió reparar la válvula mitral mediante una resección cuadrilateral de P3 asociado a una plicatura más anuloplastía con anillo, logrando un buen resultado de la reparación.

APORTE DEL ECO 3D. El Eco 3D permitió identificar y caracterizar con mayor precisión el segmento valvular que presentaba el prolapso, permitiendo predecir y programar su reparación quirúrgica.

CASO 4. Mujer de 78 años, con antecedente de recambio valvular mitral con prótesis mecánica en 1994 por enfermedad de Barlow. Sin intercurrencias hasta el año 2005 cuando consulta por cuadro infeccioso gastrointestinal asociado a bacteriemia por Enterococcus faecalis. Se amplió estudio con ETT y ETE que objetivaron adecuado funcionamiento protésico, el disco en posición posterior presentaba por su cara auricular una lesión filiforme hipermóvil de 8 x 4 mm y el anillo se encontraba engrosado de forma difusa sobre su cara auricular. Se adquirió un volumen en 3D de la prótesis que permitió un análisis mediante el recorte en distintos planos, identificando en la región perianular hacia lateral y posterior una cavidad de $16 \mathrm{~mm}$ de diámetro con expansión sistólica y material heterogéneo en su interior. Lesión compatible con un absceso vaciado (pseudoaneurisma) a la cavidad ventricular y que se encontraba en estrecha relación con la arteria circunfleja y el seno coronario (Figura 6). Se indicó cirugía cardíaca, los hallazgos del intraoperatorio confirmaron lo evidenciado por el Eco 3D, reparando el defecto con un parche de pericardio e implantando una prótesis biológica, la evolución postoperatoria fue satisfactoria.

APORTE DEL ECO 3D. El Eco 3D permitió detallar confiablemente el tamaño y relaciones anatómicas del pseudoaneurisma, otorgando valiosa información al equipo quirúrgico.

\section{Discusión:}

El diagnóstico ecocardiográfico de endocarditis requiere la presencia de lesiones macroscóspicas, dentro de ellas se encuentran las vegetaciones, que por su rápido movimiento requieren para su correcta visualización una técnica con alta resolución temporal como son el Eco 2D y el modo M. Sin embargo, dichos métodos tienen una limitada resolución espacial, aspecto donde el Eco 3D es altamente superior, permitiendo una mejor comprensión anatómica de las lesiones y sus estructuras relacionadas. Confiriendo un valor agregado al Eco 2D, especialmente cuando se 
Tabla 1. Resumen del aporte del Eco 3D en cada caso.

\begin{tabular}{|c|c|c|c|c|}
\hline CASO & LESIÓN & APORTE 3D & FASE & IMAGEN REFERENCIAL \\
\hline 1 & $\begin{array}{l}\text { Perforación velo } \\
\text { anterior válvula } \\
\text { mitral. }\end{array}$ & $\begin{array}{c}\text { Identificación } \\
\text { perforación } \\
\text { Estimar } \\
\text { posibilidad de } \\
\text { reparación } \\
\text { quirúrgica }\end{array}$ & Crónica & \\
\hline 2 & $\begin{array}{l}\text { Perforación válvula } \\
\text { aórtica bicúspide, } \\
\text { absceso de velo } \\
\text { anterior mitral }\end{array}$ & $\begin{array}{c}\text { Identificar } \\
\text { mecanismo de la } \\
\text { lesión }\end{array}$ & Aguda & \\
\hline 3 & $\begin{array}{l}\text { Rotura de cuerda y } \\
\text { prolapso de velo } \\
\text { posterior mitral } \\
\text { (P3) }\end{array}$ & $\begin{array}{c}\text { Predecir } \\
\text { posibilidad de } \\
\text { reparación }\end{array}$ & Crónica & \\
\hline 4 & $\begin{array}{c}\text { Pseudoaneurisma } \\
\text { de prótesis } \\
\text { mecánica mitral }\end{array}$ & $\begin{array}{c}\text { Identificación y } \\
\text { caracterización de } \\
\text { la lesión }\end{array}$ & Aguda & \\
\hline
\end{tabular}

trata de detectar complicaciones de la endocarditis ${ }^{4}$. Exhibiendo superioridad en evaluar; segmentos valvulares comprometidos, compromiso perianular, dehiscencias protésicas, tamaño de las vegetaciones mejorando así la predicción del riesgo embólico ${ }^{5}$ y endocarditis de cables de dispositivos intracardiacos (permitiendo reconstruir y seguir todo el trayecto del cable comprometido). Además es superior en caracterizar lesiones destructivas como abscesos y perforaciones valvulares con un alto rendimiento ${ }^{6}$. Optimizando el manejo de dichas lesiones al lograr una mejor comunicación y comprensión de la anatomía por parte del equipo de cirugía cardíaca.

Finalmente, se debe señalar que desde una perspectiva fisiopatológica la ecocardiografía realiza un diagnóstico anatómico de la endocarditis, siendo esto un hecho tardío en el curso de la enfermedad ${ }^{7}$. Por este motivo nuevas técnicas de imágenes que realizan un diagnóstico a nivel molecular y celular como son el PET-CT han sido integrados de forma complementaria a la ecocardiografía en algoritmos multi-imagen 4,5 , mejorando y anticipando el diagnóstico, especialmente en presencia de material protésico intracardiaco (marcapasos, desfibriladores, prótesis valvulares). No obstante lo anterior, seguirá siendo la ecocardiografía la piedra angular para la evaluación de pacientes con sospecha o diagnóstico de endocarditis, más aún con el apoyo de la Eco 3D, que aporta un valor agregado en todos los aspectos de la endocarditis y que se resume en la Tabla 1. 
1. DILLON JC, FEIGENBAUM H, KONECKE LL, DAVIS RH, CHANG S. Echocardiographic manifestations of valvular vegetations. Am Heart J. 1973;86:698-704.

2. HABIB G, LANCELLOTTI P, ANTUNES MJ, BONGIORNI MG, CASALTA JP, DEL ZOTTI F, et al. 2015 ESC Guidelines for the management of infective endocarditis. European Heart Journal. 2015; 36: 3075-3123.

3. VEGA J, CÓRDOVA S, MUÑOZ MC, GABRIELLI L, MCNAB P, SAAVEDRA R. Reparación de una perforación valvular mitral como secuela de endocarditis, aporte de la ecocardiografía tridimensional. Rev Chil Cardiol. 2016;35:163-168.

4. YONG MS, SAXENA P, KILLU AM, COFFEY S, BURKHART HM, WAN S-H, et al. The preoperative evaluation of infective endocarditis via 3-Dimensional transesophageal echocardiography. Texas Hear Inst J. 2015;42:372-376.

5. BERDEJO J, SHIBAYAMA K, HARADA K, TANAKA J, MI-
HARA H, GURUDEVAN S V., et al. Evaluation of vegetation size and its relationship with embolism in infective endocarditis: A Real-Time 3-Dimensional transesophageal echocardiography study. Circ Cardiovasc Imaging. 2014;7:149-154.

6. THOMPSON KA, SHIOTA T, TOLSTRUP K, GURUDEVAN $S$ V, SIEGEL RJ. Utility of three-dimensional transesophageal echocardiography in the diagnosis of valvular perforations. Am J Cardiol. 2011;107:100-102.

7. THUNY F, GAUBERT JY, JACQUIER A, TESSONNIER L, CAMMILLERI S, RAOULT D, et al. Imaging investigations in infective endocarditis: Current approach and perspectives. Arch Cardiovasc Dis. 2013;106:52-62.

8. WONG D, RUBINSHTEIN R, KEYNAN Y. Alternative cardiac imaging modalities to echocardiography for the diagnosis of infective endocarditis. Am J Cardiol. 2016;118:1410-1418.

9. MILLAR BC, HABIB G, MOORE JE. New diagnostic approaches in infective endocarditis. Heart. 2016;102:796-807. 